\title{
THE COMPARATIVE ANALYSIS OF THE TERM STRUCTURE MODELS OF THE AFFINE YIELD CLASS
}

\author{
Natalia G. Ilieva \\ Faculty of Mathematics and Informatics, University of Sofia \\ 5 Jeames Baucher, room 303, 1126 Sofia, Bulgaria \\ Tel: (3592) 6256543. Fax: (3592) 739941 \\ iliev@itwm.uni-kl.de
}

\begin{abstract}
There are some various methods of construction of models of a change of the short interest rate for the analysis of a term structure of an affine yield class that have been introduced by Brown and Schaefer (1991). In the present paper the analysis of three from them is carried out the V-model (Vasiček, 1977), CIR-model (Cox, Ingersoll and Ross, 1985), and MC-model (Medvedev and Cox, 1996). The main attention is given to a research of mutual properties of functions of affine structure of these models and properties of probability distributions of stochastic processes, which in these models the short interest rate follows. The analysis of the term structure of affine yield class in the literature is usually carried out under the "adjusted by risk" equivalent martingal probability measure. In this paper the analysis is made under objective probability measure. The functions of affine structure and the probability distributions of the short interest rate are obtained in the explicit form. Usually in the literature the V-model and CIR-model are considered as the models of different classes. In the paper these models together with a $\mathrm{MC}$-model are set as the models of one class. The unity of models is the fact that the process of the short interest rate is described by the same scheme with the lower reflecting boundary which in the V-model is deleted to a minus infinity, it is equal to zero in the CIR-model, and in the MC-model this boundary takes an intermediate position. It is shown that the short interest rate in a transient regime is the sum of two components: 1) random variable that is subjected by shifted gamma distribution; 2) random variable that has a compound Poisson distribution with an exponential distribution of summands. Under transition to the stationary regime the second component disappears. If the value of the shift parameter in the first component is increased ad infinitum then the short interest rate is described by the $\mathrm{V}$-model. If the significance of a shift parameter in the first component is equal to zero then the short interest rate is described by the CIR-model. If the shift parameter in the first component takes an intermediate position then the short interest rate is described by the MC-model. Under such modification of a shift parameter from zero ad infinitum for fixed expectation and variance of process of the short interest rate its marginal distribution is modified from the gamma distribution to the normal distribution.
\end{abstract}

Keywords: short interest rate, bond price, term structure model, affine yield class, stochastic process, reflecting boundary, compound Poisson distribution. 


\section{Introduction}

The affine yield class of term structure models for the first time was introduced by Brown and Schaefer (1991) which noted that some models of term structure have identical closed form. They show that this takes place if the drift and the square of volatility of the short interest rate are linear functions of the short interest rate and the drift and the volatility are independent on time. Duffie and Kan (1993) have extended these ideas to a multiple factor setting. The examples of such models among the single factor models are the V-model (Vasiček, 1977), CIR-model (Cox, Ingersoll, and Ross, 1985), and MCmodel (Medvedev and Cox, 1996).

Usually in the literature the V-model and CIR-model are considered as the models of different classes. In this paper the base is found that these models together with a MCmodel could be considered as the models of one class. This base is the fact that the process of the short interest rate is described by on and the same scheme, but with different lower reflecting boundaries. This boundary in the $\mathrm{V}$-model is se to be minus infinity, it is equal to zero in the CIR-model, and in the MC-model this boundary takes an intermediate position.

The main attention is given to an investigation of mutual properties of functions of affine structure of these models and probability properties of stochastic processes, which the short interest rate follows in these models. The analysis is made under objective probability measure. The functions of the affine structure, the probability distributions, and the moment generating functions of the short interest rate are obtained in the explicit form.

It is shown that the short interest rate in the transient regime is a sum of two components: 1) random variable that is subjected by shifted gamma distribution; 2) random variable that has a compound Poisson distribution with an exponential distribution of summands. Under transition to the stationary regime, the second component disappears. If the value of the shift parameter in the first component is increased ad infinitum then the short interest rate is described by the V-model. If the significance of the shift parameter in the first component is equal to zero then the short interest rate is described by the CIRmodel. If the shift parameter in the first component takes an intermediatc position then the short interest rate is described by the MC-model. Under such modification of a shift parameter from zero ad infinitum for fixed expectation and variance of process of the short interest rate its marginal distribution is modified from the gamma distribution to the normal distribution.

The outline of the paper is as follows. Some necessary determinations and known relations are given in introduction below. Section 1 contains the results concerning functions of affine structure. Section 2 is devoted to deriving the probability density functions and the moment generating functions of the short interest rate and investigation of their properties. 
Consider zero-coupon bond with a face value 1 and date of maturity $T$ provided that the risk-free short interest rate at time instant $t<T$ is equal to $r$.If its price can be expressed as

$$
P(t, r, T)=\exp \{A(t, T)-r B(t, T)\}
$$

then one speaks, that the term structure model of interest rate belongs to an affine yield class (Brown and Schaefer, 1991; Duffie, 1992). Let's note, that is in this case the yield rate determined by the formula

$$
y(t, r, T)=[r B(t, T)-A(t, T)] /(T-t) .
$$

Thus, this class is called affine class due to the fact, that the yield interest rate $y(t, r, T)$ linearly depends on value of the short interest rate $r=r(t)$. Various aspects of properties of affine models were considered in the various articles (see for example, Brown and Schaefer, 1994; Rogers, 1995).Functions $A(t, T)$ and $B(t, T)$ are called below functions of an affine term structure. These functions are determined from the so-called partial differential equation for a term structure (the fundamental partial differential equation of security pricing) under no arbitrage condition

$$
P_{t}+[\mu(t, r)+\lambda(t, r) \sigma(t, r)] P_{r}+\mathrm{S} \sigma^{2}(t, r) P_{r r}-r P=0,
$$

where the arguments of function $P(t, r, T)$ are omitted for brevity, and the partial derivatives on variables $t$ and $r$ are denoted by appropriate subscript. A boundary condition of the equation ( 3 ) is given by

$$
P(T, r, T)=1 \text { for any of } r \text {. }
$$

The functions $\mu(t, r)$ and $\sigma(t, r)$ in (3) are the drift and the volatility of the short interest rate $r(t)$.The latest follows a diffusion process described by the stochastic differential equation

$$
d r=\mu(t, r) d t+\sigma(t, r) d W(t)
$$

The function $\lambda(t, r)$ is a market price of risk and it is set exogenous as well as the functions $\mu(t, r)$ and $\sigma(t, r)$. The price $P(t, r, T)$ also follows a stochastic diffusion process. Under no arbitrage condition, the drift of instantaneous rate of return of the bond is determined by expression $\mu(t, r)+\lambda(t, r) \sigma(t, r)$. 
In order the solution of the equation (3) to have a form given by (1), it is enough, $t$ the functions $\mu(t, r)+\lambda(t, r) \sigma(t, r)$ and $\sigma^{2}(t, r)$ to be affine, i.e. linear with respect to $r$. This can be achieved if one sets (Björk, 1995)

$$
\begin{aligned}
& \mu(t, r)=\alpha(t) r+\beta(t), \\
& \sigma^{2}(t, r)=\gamma(t) r+\delta(t), \\
& \lambda(t, r) \sigma(t, r)=\xi(t) r+\eta(t) .
\end{aligned}
$$

Hereinafter for brevity we shall refer to $\alpha(t), \beta(t), \gamma(t), \delta(t), \xi(t)$ and $\eta(t)$ as coefficients of an affine structure. Substituting (1), (6)-(8) in equation (3), accounting for boundary condition (4), and considering $t$ and $r$ as independent variables, one obtains the following two equations:

$$
\begin{aligned}
& \frac{d B}{d t}=\mathrm{S} \gamma(t) B^{2}(t, T)-[\alpha(t)+\xi(t)] B(t, T)-1, \quad B(T, T)=0 \\
& \frac{d A}{d t}=-\mathrm{S} \delta(t) B^{2}(t, T)+[\beta(t)+\eta(t)] B(t, T), \quad A(T, T)=0 .
\end{aligned}
$$

$A(t, T)$ can be obtained by integrating (10) after $B(t, T)$ is found as a solution of (9).

$$
A(t, T)=\int_{t}^{T}\left[-\frac{1}{2} \delta(s) B^{2}(s, T)+\{\beta(s)+\eta(s)\} B(s, T)\right] d s .
$$

The equation (9) is the Riccati equation and in general it has no explicit solution. However in one important special case it is possible to derive such a solution.

Lemma. Let there exist a constant $c \neq 0$, such that the following equality is satisfied:

$$
\frac{c^{2}}{2} \gamma(t)-c[\alpha(t)+\xi(t)]-1=0,
$$

Then the solution of the equation (9) has the form

$$
B(t, T)=\frac{c J(t, T)}{c+J(t, T)}
$$


where it the following notation in introduced:

$$
J(t, T)=\int_{t}^{T} \exp \left\{\int_{t}^{y}\left(\frac{2}{c}+\alpha(x)+\xi(x)\right) d x\right\} d y .
$$

The proof of this statement follows from results given in item 4.9 (b) of chapter 1 of Kamke (1959), and it is easy for checking up by a direct substitution of (13) - (14) in the equation (9).

Let's note that the formal condition (12) can be interpreted as follows sense. If the function of an affine structure $B(t, T)$ has steady-state level (i.e. it tends to some constant when the difference $T-t$ tends to $\infty$ ), then the condition (12) takes place with a positive constant $c=\lim _{t \rightarrow-\infty} B(t, T)=c$. Indeed, if we substitute the limiting value $B(-\infty, T)=c=$ const in the equation (9), then the left-hand side of this equation turns to zero, and the right-hand side (9) transforms in condition (12).

\section{Affine term structure with constant coefficients}

Let's assume, that the coefficients of an affine term structure $\alpha, \beta, \gamma, \delta$, $\xi$ and $\eta$ are constants. The models of a term structure are in this case referred to as time homogeneous ones (Brown and Schaefer, 1994). Before starting the to analytical consideration of this case, we shall comment on the meaning of these coefficients.

Let's make the natural assumption that there exist a stationary regime of the short interest rate process $r(t)$. That is, with the course of time the variance of process $r(t)$ can not be infinitely increased, and it tends to a some finite level. In this case parameter $\alpha$ should be negative, and its absolute value determines the speed of adjustment to the stationary regime (that is it is equivalent to the choice of a time scale).The stationary regime is reached faster when $\alpha$ is small. For convenience, sometimes we will use notation $k=-\alpha, k>0$.

The expectation of process $r(t)$ is determined by the equation $E[\mu(t, r)]=\alpha E[r(t)]$ $+\beta=0$ in the stationary regime. Hence the sense of parameter $\beta$ is seen. Denote $E[r]=\theta$ in the stationary regime. Then we have $\beta=-\alpha \theta=k \theta$. Because the average value of the short interest rate should be positive, $\beta>0$.A process of the short interest rate $r(t)$ that has such properties usually is known as the mean reverting process, or Ornstein-Uhlenbeck process.

The parameters $\gamma$ and $\delta$ together with values $r(t)$ determine square of volatility of the short interest rate, as it is seen from equality (7). An inequality $\gamma r+\delta \geq 0$ for any $r$ therefore should held. Rewriting this inequality in the form $r \geq-\delta / \gamma$, one sees that the 
value $(-\delta / \gamma)$ should be considered as a lower bound of possible values of the short interest rate. Because, values of $r$ are positive, this inequality is fulfilled for example, when the parameters $\gamma$ and $\delta$ are non-negative, i.e. $\gamma \geq 0$ and $\delta \geq 0$.

As it follows from (8), parameters $\xi$ and $\eta$ together with values of process $r(t)$ and volatility $\sigma(t, r)$ determine a market price of the risk. It is natural to consider the interest rate and volatility as non-negative values. At the same time, in order the risk premium to be positive, it is necessary that the values $\lambda(t, r)$ be positive. Therefore we can consider parameters $\xi$ and $\eta$, i.e. $\xi \geq 0$ and $\eta \geq 0$.

We will use the lemma above to determine the function $B(t, T)$. If the coefficients of an affine structure are constant then equality (12) can be considered as the equation for constant $c$ and we can write it as

$$
\gamma c^{2}-2(\alpha+\xi) c-2=0
$$

When $\gamma \geq 0$ this equation have real roots. For $\gamma>0$ the roots have the form

$$
c_{+}=\frac{1}{\gamma}\left(\alpha+\xi+\sqrt{(\alpha+\xi)^{2}+2 \gamma}\right), c_{-}=\frac{1}{\gamma}\left(\alpha+\xi-\sqrt{(\alpha+\xi)^{2}+2 \gamma}\right) .
$$

Let's for brevity denote $\varepsilon=\sqrt{(\alpha+\xi)^{2}+2 \gamma}>0$. Hence, the integral $J(t, T)$ is calculated from formula (14) using these roots:

$$
J_{+}(t, T)=\frac{1}{\varepsilon}\left(e^{\varepsilon(T-t)}-1\right), \quad J_{-}(t, T)=\frac{1}{\varepsilon}\left(1-e^{-\varepsilon(T-t)}\right) .
$$

Substitution of values $c_{+}$and $J_{+}(t, T)$ in expression (13) gives an expression for the function $B(t, T)$ in the form

$$
B(t, T)=\frac{2\left(e^{\varepsilon(T-t)}-1\right)}{2 \varepsilon+(\varepsilon-\alpha-\xi)\left(e^{\varepsilon(T-t)}-1\right)} .
$$

The substitution of values $c_{-}$and $J_{-}(t, T)$ in (13) results in a function for $B(t, T)$ distinguishing from (15) only by the opposite sign. However negative function $B(t, T)$ contradicts to economic sense of the price (1), because with the increase of the interest rate $r$ the bond price will increase in this case, too. Therefore this version can not be accepted and the unique solution of (9) is given by (15).

As it is natural to expect in the case of constant coefficients of an affine structure, the function $B(t, T)$ does not depend on absolute values two variables $t$ and $T$ but it 
depends on their difference $T-t$. That is $B(t, T)$ is a function of one argument. Let's denote $\tau=T-t$. Then we have $\frac{d B(T-t)}{d t}=-\frac{d B(\tau)}{d \tau}$. The equation (9) can be written in this case as

$$
\frac{d B}{d \tau}=1+(\alpha+\xi) B(\tau)-\frac{1}{2} \gamma B^{2}(\tau), \quad B(0)=0, \tau \geq 0
$$

Rewriting this equation in the symmetrical form, we have

$$
d \tau-\frac{d B(\tau)}{1+(\alpha+\xi) B(\tau)-0,5 \gamma B^{2}(\tau)}, \quad \tau \geq 0 .
$$

The following integral relation follows from equation (17)

$$
\tau=\int_{0}^{B(\tau)} \frac{d B}{1+(\alpha+\xi) B-0,5 \gamma B^{2}}, \quad \tau \geq 0,
$$

it is useful in calculation of the function $A$.

Now we address the equation (10) for function $A(t, T)$ of an affine term structure. The right-hand side of the equation (10) depends on a variable $t$ only through function $B$. Therefore function $A(t, T)$ will depend only on the difference of the arguments as well, that is $A(t, T)=A(T-t)=A(\tau)$. Again, using equality $\frac{d A(T-t)}{d t}=-\frac{d A(\tau)}{d \tau}$, it is possible to write the equation (10) in the symmetrical form as

$$
d \tau=\frac{d A(\tau)}{0,5 \delta B^{2}(\tau)-(\beta+\eta) B(\tau)}, \quad A(0)=0, \tau \geq 0 .
$$

Combining equations (17) and (19) we obtain an integral relation for function $A(\tau)$

$$
A(\tau)=\int_{0}^{B(\tau)}\left(\frac{0,5 \delta B^{2}-(\beta+\eta) B}{1+(\alpha+\xi) B-0,5 \gamma B^{2}}\right) d B
$$

The advantage of relation (20) compare to (11) is that (11) is an integral of a rather composite function on variable $\tau$, while $(20)$ is an integral from a simple rational 
expressions of variable $B$. Actually, (20) is a tabulated integral. Let's introduce for brevity the notations

$$
\begin{array}{ll}
\varepsilon=\sqrt{(\alpha+\xi)^{2}+2 \gamma,} & \vartheta_{+}=\varepsilon+(\alpha+\xi), \\
\omega=\gamma(\beta+\eta)-\delta(\alpha+\xi), & \vartheta=\varepsilon-(\alpha+\xi) .
\end{array}
$$

Then the integral (20) can be represented in one of the following equivalent expressions

$$
\begin{aligned}
& A(\tau)=\frac{\delta}{\gamma}[\tau-B(\tau)]-\frac{\omega}{\gamma^{2}}\left[(\alpha+\xi) \tau-\ln \left(1+(\alpha+\xi) B(\tau)-\frac{\gamma}{2} B^{2}(\tau)\right)\right] \\
& A(\tau)=\frac{\delta}{\gamma}[\tau-B(\tau)]+\frac{\omega}{\varepsilon \gamma^{2}}\left[\vartheta_{-} \ln \left(1+\frac{\vartheta_{+}}{2} B(\tau)\right)+\vartheta_{+} \ln \left(1-\frac{\vartheta_{-}}{2} B(\tau)\right)\right], \\
& A(\tau)=\frac{\delta}{\gamma}[\tau-B(\tau)]-\frac{\omega}{\gamma^{2}}\left[\vartheta_{+} \tau-2 \ln \left(1+\frac{\vartheta_{+}}{2} B(\tau)\right)\right] \\
& A(\tau)=\frac{\delta}{\gamma}[\tau-B(\tau)]+\frac{\omega}{\gamma^{2}}\left[\vartheta_{-} \tau+2 \ln \left(1-\frac{\vartheta_{-}}{2} B(\tau)\right)\right]
\end{aligned}
$$

Substituting the explicit expression of function $B(\tau)(15)$ in these equalities, we obtain the explicit expressions for function $A(\tau)$ in term maturity $\tau$. On the other hand, from an integrated relation (18) it is possible to obtain the following expression

$$
\tau=\frac{1}{\varepsilon}\left[\ln \left(1+\frac{\vartheta_{+}}{2} B(\tau)\right)-\ln \left(1-\frac{\vartheta_{-}}{2} B(\tau)\right)\right] .
$$

Using (26), one can express function $A(\tau)$ as a function of $B(\tau)$ only.

Let's note that the functions of an affine term structure for the considered case in form (15) and (25) were derived earlier (see Medvedev and Cox, 1996). Hercinafter we shall mention this model of an affine term structure as a MC-model. Let's bring also two famous special cases, which frequently are used for analysis of the short interest rates and the yield rates.

When volatility and market price of risk do not depend on values of the short interest rate $r$, that is $\gamma=0$ and $\xi=0$, we obtain Vasiček model (Vasiček, 1977). In this case $\varepsilon=|\alpha|$ and we have 


$$
\begin{aligned}
& B(\tau)=\frac{1}{|\alpha|}\left(1-e^{-|\alpha| \tau}\right)=\frac{1}{\alpha}\left(e^{\alpha \tau}-1\right), \quad \tau \geq 0 ; \\
& A(\tau)=\frac{1}{\alpha}\left(\frac{\delta}{2 \alpha}+\beta+\eta\right)[\tau-B(\tau)]+\frac{\delta}{4 \alpha} B^{2}(\tau), \quad \tau \geq 0 .
\end{aligned}
$$

Formula (28) can be derived from equalities (22)-(25) as a limit case $\gamma \rightarrow 0$.However, the calculation of limits might be complicated, and an easier way to derive (28) is to set $\gamma=0$ and $\xi=0$ in (20).

In the other special case it is supposed, that $\delta=0$ and $\eta=0$. This version of the term structure model of the interest rates is usually mentioned as the CIR-model (Cox, Ingersoll, and Ross, 1985). In this case function $B(\tau)$ docs not become simpler and has a form (15) and the function $A(\tau)$ gets the form

$$
A(\tau)=\frac{\beta}{\varepsilon \gamma}\left[\vartheta_{+} \ln \left(1-\frac{\vartheta_{-}}{2} B(\tau)\right)+\vartheta_{-} \ln \left(1+\frac{\vartheta_{+}}{2} B(\tau)\right)\right],
$$

when equality (23) is used, or

$$
A(\tau)=\frac{\beta}{\gamma}\left[\vartheta_{-} \tau+2 \ln \left(1-\frac{\vartheta_{-}}{2} B(\tau)\right)\right], \quad \tau \geq 0,
$$

when equality (25) is used.

Let's note that the following equalities, that obtained with the help of expression (15), take place

$$
\begin{aligned}
& \ln \left(1+\frac{\vartheta_{+}}{2} B(\tau)\right)=\varepsilon \tau-\ln \left(1+\frac{\vartheta_{-}}{2 \varepsilon}\left(e^{\varepsilon \tau}-1\right)\right), \\
& \ln \left(1-\frac{\vartheta_{-}}{2} B(\tau)\right)=-\ln \left(1+\frac{\vartheta_{-}}{2 \varepsilon}\left(e^{\varepsilon \tau}-1\right)\right) .
\end{aligned}
$$

Using these equalities, it is possible to derive the explicit expression $A$ ( $\tau$ ) from (29) or (30) in term $\tau$ as follows 


$$
A(\tau)=\frac{\beta \vartheta_{-}}{\gamma} \tau-\frac{2 \beta}{\gamma} \ln \left(1+\frac{\vartheta_{-}}{2 \varepsilon}\left(e^{\varepsilon \tau}-1\right)\right)=\frac{2 \beta}{\gamma} \ln \left(\frac{2 \varepsilon e^{\vartheta_{-} \tau / 2}}{2 \varepsilon+\vartheta_{-}\left(e^{\varepsilon \tau}-1\right)}\right)
$$

The above form was obtained earlier by Cox, Ingersoll, and Ross (1985).

Let's note that between analytical expressions for function $A(\tau)$ from $\mathrm{MC}$-model and from CIR-model, respectively, are related as follows:

$$
A_{M C}(\tau)=\frac{\delta}{\gamma}[\tau-B(\tau)]-\frac{\omega}{\gamma \beta} A_{C I R}(\tau)
$$

For fixed values of coefficients of the affine structure $\alpha, \beta, \gamma, \delta$, it is possible to set also other useful relation between functions $A(\tau)$ and $B(\tau)$ of various models of the short interest rate. For this purpose, we shall supply functions with subscripts corresponding to respective models, as it is already made in the formula (34. The following propositions hold.

Proposition 1.The function $B(\tau)$ defined by formula (15) is monotonically increasing convex up function that has a horizontal asymptote $\lim _{\tau \rightarrow \infty} B(\tau)=2 /(\varepsilon-\alpha-\xi)$. Also the inequalities $0 \leq B(\tau) \leq-1 / \alpha=B_{V}(\infty)$ are valid for any $\tau>0$.

Proof. The formula (15) can be written as

$$
B(\tau)=\left(\frac{\varepsilon}{e^{\varepsilon \tau}-1}+\frac{\varepsilon-\alpha-\xi}{2}\right)^{-1}
$$

Twice differentiating this function, we see that the first derivative is positive and the second derivative is negative. It proves the first part of the proposition.

Proposition 2. Functions $A$ ( $\tau$ ) determined by the formulae (22) - (25), (28), (29) (30), (33) have sloping asymptotes that are given by straight lines $a \tau+b$. The values of

\begin{tabular}{|c|c|c|c|}
\hline & V-model & CIR-model & MC-model \\
\hline$a$ & $\frac{\beta+\eta}{\alpha}+\frac{\delta}{2 \alpha^{2}}$ & $-\frac{\beta \vartheta_{+}}{}$ & $\frac{\delta}{\gamma}+\frac{\omega \vartheta_{+}}{\gamma^{2}}$ \\
\hline$b$ & $\frac{\beta+\eta}{\alpha^{2}}+\frac{3 \delta}{4 \alpha^{3}}$ & $\frac{2 \beta}{\gamma} \ln \left(\frac{2 \varepsilon}{\vartheta_{-}}\right)$ & $-\frac{2 \delta}{\gamma \vartheta_{-}}-\frac{2 \omega}{\gamma^{2}} \ln \left(\frac{2 \varepsilon}{\vartheta_{-}}\right)$ \\
\hline
\end{tabular}
coefficients $a$ and $b$ for various models of the short interest rates are given in the following table 
Proposition 3. The analytical properties of functions $A(\tau)$ for various models of the short interest rate are characterized by the following table

\begin{tabular}{|c|c|c|c|}
\hline Behavior of $A(\tau)$ & $\mathrm{V}$ - model & CIR-model & MC-model \\
\hline $\begin{array}{l}\text { Monotone } \\
\text { decrease }\end{array}$ & $-\frac{1}{2 \alpha} \leq \frac{\beta+\eta}{\delta}$ & $\begin{array}{c}\text { for any } \\
\alpha<0, \gamma>0, \xi \leq 0\end{array}$ & $\frac{1}{\vartheta_{-}} \leq \frac{\beta+\eta}{\delta}$ \\
\hline Convexity up & $-\frac{1}{\alpha}<\frac{\beta+\eta}{\delta}$ & $\begin{array}{c}\text { for any } \\
\alpha<0, \gamma>0, \xi \leq 0\end{array}$ & $\frac{2}{\vartheta_{-}}<\frac{\beta+\eta}{\delta}$ \\
\hline $\begin{array}{l}\text { Existence } \\
\text { of } \\
\text { minimum }\end{array}$ & $0 \leq \frac{\beta+\eta}{\delta} \leq-\frac{1}{2 \alpha}$ & no & $0 \leq \frac{\beta+\eta}{\delta} \leq \frac{1}{\vartheta_{-}}$ \\
\hline $\begin{array}{c}\text { Point } \\
\text { of } \\
\text { inflection }\end{array}$ & $0 \leq \frac{\beta+\eta}{\delta} \leq-\frac{1}{\alpha}$ & no & $0 \leq \frac{\beta+\eta}{\delta} \leq \frac{2}{\vartheta}$ \\
\hline $\begin{array}{l}\text { Monotone } \\
\text { increase }\end{array}$ & $\beta+\eta<0$ & no & $\beta+\eta<0$ \\
\hline
\end{tabular}

The minimum of function $A(\tau)$ and its point of inflection, if any, are reached for the values of variable $\tau$ given in the table

\begin{tabular}{ccc}
\hline $\begin{array}{c}\text { Point } \\
\text { of } \\
\text { minimum } \\
\text { Point } \\
\text { of } \\
\text { inflection }\end{array}$ & $\frac{1}{\alpha} \ln \left(1+2 \alpha \frac{\beta+\eta}{\delta}\right)$ & $\frac{1}{\varepsilon} \ln \left(\frac{\delta+(\beta+\eta) \vartheta_{+}}{\delta-(\beta+\eta) \vartheta_{-}}\right)$ \\
\hline
\end{tabular}

The propositions $1-3$ can be proved by the usual technique of calculus. In general, it is sufficient to calculate first and second derivatives of functions $A(\tau)$ and $B(\tau)$ and investigate their behavior. These proofs are omitted here. 


\section{Probability properties of the short interest rate processes of affine class}

If the functions of drift $\mu(t, r)$ and volatility $\sigma(t, r)$ of the short interest rate $r(t)$ in the equation (3) are differentiable with respect to second argument, the probability density function $f(r, t)$ of process $r(t)$ is determined by the equation

$$
f_{r}(r, t)=1 / 2\left[\sigma^{2}(t, r) f(r, t)\right]_{r r}-[\mu(t, r) f(r, t)]_{r} .
$$

Here as before the subscripts denote partial derivative with respect to appropriate variable. The equation (35) is known as the forward Kolmogorov equation in theory of stochastic processes and as Fokker-Plank equation in diffusions theory(see for example, Lamperti, 1977). In the case of constant coefficients of affine structure, the equation (35) takes the form

$$
f_{t}(r, t)=1 / 2[(\gamma r+\delta) f(r, t)]_{r r}-[(\alpha r+\beta) f(r, t)]_{r}
$$

Let's introduce the following notations:

$g(x \mid q, c)=\frac{c^{q+1} x^{q}}{\Gamma(q+1)} e^{-c x}-$ the probability density function of gamma distribution with a shape parameter $(q+1)$ and a scale parameter $c$, where $x \geq 0$.

$p(j \mid u)=\frac{u^{j}}{j !} e^{-u}-$ the Poisson distribution with a parameter $u>0, \mathrm{j}=0,1,2, \ldots$

$b(j \mid q, \theta)=\frac{\Gamma(q+j)}{j ! \Gamma(q)} v^{j}(1-v)^{q}-$ the negative binomial probability distribution with parameters $q>0, v \in(0,1), j=0,1,2, \ldots$

Theorem 1. If $\gamma r+\delta>0$, i.e. $r \in(-\delta / \gamma, \infty)$, then the conditional probability density function $f(r, t \mid r(s)=R), s<t$, which gives solution of the equation (36), has a form

$$
\begin{array}{r}
f(r, t \mid r(s)=b)=\sum_{j=0}^{\infty} \frac{u^{j}}{j !} e^{-u} \times \frac{c^{q+j+1}(r+\delta / \gamma)^{q+j}}{\Gamma(q+j+1)} e^{-c(r+\delta / \gamma)}= \\
=\sum_{j=0}^{\infty} p(j \mid u) \times g(r+\delta / \gamma \mid q+j, c), \quad-\delta / \gamma<r<\infty,
\end{array}
$$

where additionally the following notations are used 


$$
u=c(R+\delta / \gamma) e^{\alpha(t-s)}, \quad c=\frac{2 \alpha}{\gamma}\left(e^{\alpha(t-s)}-1\right)^{-1}, \quad q=2 \frac{\gamma \beta-\alpha \delta}{\gamma^{2}}-1
$$

The joint probability density function $f(r, t ; R, s), s<t$, of values of the short interest rate $r$ $(t)=r, r(s)=R$ has a representation

$$
\begin{array}{r}
f(r, t ; R, s)=\sum_{j=0}^{\infty} b\left(j \mid q+1, e^{\alpha(t-s)}\right) \times g\left(r+\frac{\delta}{\gamma \mid} q+j, c\right) \times g\left(R+\frac{\delta}{\gamma} \mid q+j, c\right), \quad \text { (39) } \\
-\delta / \gamma<r<\infty, \quad-\delta / \gamma<R<\infty .
\end{array}
$$

Under the same conditions, the marginal probability density function $f(r, t)$ of this joint density is a density of shifted gamma distribution with the shift parameter $(-\delta \gamma)$, the form parameter $(q+1)$, and a scale parameter $c_{0}>0$, given by

$$
f(r, t)=\frac{c_{0}^{q+1}(r+\delta / \gamma)^{q}}{\Gamma(q+1)} e^{-c_{0}(r+\delta / \gamma)}, \quad-\delta / \gamma<r<\infty,
$$

where $c_{0}=\lim _{(t s) \rightarrow \infty} \frac{2 \alpha}{\gamma}\left(e^{\alpha(t-s)}-1\right)^{-1}=-\frac{2 \alpha}{\gamma}, \alpha<0$.

Proof. The equation (36) in the case $\delta=0, \gamma>0,0<r<\infty$ has been considered by Feller (1951), which has shown, that the conditional probability density $f(r, t \mid r(s)=R), s$ $<t$, has the form (in the interpretation of Cox, Ingersoll and Ross, 1985)

$$
f(r, t \mid r(s)=R)=c e^{-u-v}\left(\frac{v}{u}\right)^{q / 2} I_{q}(2 \sqrt{u v})
$$

where $c=\frac{2 \alpha}{\gamma\left(e^{\alpha(t-s)}-1\right)}, u=c R e^{\alpha(t-s)}, v=c r, q=\frac{2 \beta}{\gamma}-1$, and $I_{q}$ (.)is the modified Bessel function of the first kind of order $q$. The density (41) is a density of the noncentral chi-square probability density function with $(2 q+2)$ degrees of freedom and parameter of noncentrality $2 u$. Let's note, that when $t-s \rightarrow \infty$, the density (41) converges to the density of gamma distribution with form parameter $2 \beta / \gamma$ and scale parameter $2|\alpha| / \gamma$.

The Feller method can be used in more general case when $\delta \neq 0, \gamma r+\delta>0$, i. e. $-\delta / \gamma<r<\infty$. Indeed, let's set a new variable $x=r+\delta / \gamma$ in the equation (36) and let us 
introduce new parameters: $\widetilde{a}=\gamma / 2>0, \tilde{b}=\alpha, \widetilde{c}=\beta-\alpha \delta / \gamma$. Then the conditional density $\tilde{u}(x, t)=f(x-\delta / \gamma, t \mid r(s)=R), s<t$, will satisfy the equation

$$
\tilde{u}_{t}=[\tilde{a} x \tilde{u}(x, t)]_{x x}-[(\tilde{b} x+\widetilde{c}) \tilde{u}(x, t)]_{x}, \quad 0<x<\infty .
$$

This equation completely coincides with the equation considered by Feller. Therefore, solution of the equation (36) will be the same as the Feller's solution. However, variables $u, v$ and a parameter $q$ have to be understand now in another way:

$$
u \equiv c(R+\delta / \gamma) e^{\alpha(t-s)}, v \equiv c(r+\delta / \gamma), q \equiv 2(\gamma \beta-\alpha \delta) / \gamma^{2}-1
$$

Let's give one more interpretation for the obtained density. The modified Bessel functions $I_{q}(x)$ can be represented as a series

$$
I_{q}(x)=\sum_{j=0}^{\infty} \frac{(x / 2)^{2 j+q}}{j ! \Gamma(j+q+1)} .
$$

We use this expansion in expression (41) and transform it in order to represent each term of expansion as a product of two multiplicands, one of which would depend on variable $R$, and other would depend on variable $r$. This allows us to obtain conditional probability density as (37) taking in to account (38).

When $(t-s)$ is increases, the dependence between $r(t)=r$ and $r(s)=R$ disappears. Therefore, the unconditional (marginal) probability density (40) can be obtained as a limit

$$
f(r, t)=\lim _{(t-s) \rightarrow \infty} f(r, t \mid r(s)=R)=g\left(r+\delta / \gamma \mid q+1, c_{0}\right),
$$

which is exactly the probability density $(40)$.

By definition the joint probability density is expressed as

$$
f(r, t ; R, s)=f(r, t \mid r(s)=R) \times f(R, s) .
$$

Using expression (37) for $f(r, t \mid r(s)=R$ ) and expression (40) for $f(R, s)$, and making necessary transformations in expression

$$
\sum_{j=0}^{\infty} p(j \mid u) \times g(r+\delta / \gamma \mid q+j, c) \times g\left(R+\delta / \gamma \mid q+1, c_{0}\right)
$$


one can derive representation (39).

Theorem 2 . The moment generating function of conditional probability density (34) can be expressed in the form

$$
M_{r}(\tau \mid u)=\left(e^{-\delta \tau / \gamma}\left(\frac{c}{c-\tau}\right)^{q+1}\right) \exp \left\{\frac{u \tau}{c-\tau}\right\}, \quad \tau<c
$$

The moment generating function of joint probability density (39) has the form:

$$
M_{r, R}(x, y)=e^{-(x+y) \delta / \gamma}\left(\frac{1-e^{\alpha(t-s)}}{\left(1-\frac{x}{c}\right)\left(1-\frac{y}{c}\right)-e^{\alpha(t-s)}}\right)^{q+1}
$$

This generating function exists in area that is determined by the following inequality

$$
(1-x / c)(1-y / c)>e^{\alpha(t-s)} .
$$

The moment generating function of marginal probability density (40) is determined by the expression

$$
M_{r}(\tau)=e^{-\delta \tau / \gamma}\left(\frac{c_{0}}{c_{0}-\tau}\right)^{q+1} \quad, \quad \tau<c_{0}
$$

The parameters of generating functions are determined in just the same way as in the previous theorem.

Proof. The formula (44) is obvious, because it is the usual formula for a moment generating function of shifted gamma distribution (40). For the proof of the formula (43) it is enough to remember that due to the properties of gamma distribution, it is possible to consider a probability density $g(x \mid q+j, c)$ as a convolution of probability densities $g(x \mid$ $q, c)$ and $g(x \mid j-1, c)$. Therefore expression (37) can be considered as a convolution of a probability densities $g(x \mid q, c)$ and probability densities that has the form

$$
e^{-u} \delta(x)+\sum_{j=1}^{\infty} \frac{u^{j}}{j !} e^{-u} \times \frac{c(c x)^{j-1}}{\Gamma(j)} e^{-c x}
$$


The probability density (45) is a probability density of the sum of a random sum of $N$ independent and identically distributed random variables. The random number $N$ is distributed according to the Poisson law with a parameter $u$, and its value in our case is proportional to the value of the interest rate $r(s)$ at time $s$. The terms of the sum have an exponential distribution with a parameter $c$. Let's remark, that the sum of $j$ such values has a probability density of gamma distribution $g(x \mid j-1, c)$. Such distribution is known as a compound Poisson distribution (CPD) (about CPD see, for example, Gerber, 1979). The moment generating function of CPD has a form $\exp \{u[m(\tau)-1]\}$, where $u$ is a Poisson parameter, and $m(\tau)$ - the moment generating function of the terms. In our case, $m(\tau)$ is a moment generating function of an exponential distribution with a parameter $c$, that is $\quad m(\tau)=\frac{c}{c-\tau}$. Thus the probability density (37) is a probability density of a random variable that is sum of two independent random variables, one of which has a probability density $g(x \mid q, c)$, and other has a density of compound Poisson distribution (45). It is also expressed by the formula (42), taking account that in our case the gamma distribution is shifted, that is $x=r+\delta / \gamma$.

To prove formula (43) we use the definition of two-dimensional moment generating function for density (39), supposing that variables $x$ and $y$ satisfy the inequality $(1-x / c)(1 \cdot y / c)>e^{\alpha(t-s)}, s<t$,

$$
\begin{aligned}
& M_{r, R}(x, y)=E\left\{e^{r x+R y}\right\}= \\
& =e^{-(x+y) \delta / \gamma} \sum_{j=0}^{\infty}\left(\frac{c}{c-x}\right)^{q+j+1}\left(\frac{c}{c-y}\right)^{q+j+1} \frac{\Gamma(q+j+1)}{j ! \Gamma(q+1)}\left(1-e^{\alpha(t-s)}\right)^{q+1} e^{\alpha(t-s) j}= \\
& =e^{-(x+y) \delta / \gamma}\left(\frac{c^{2}\left(1-e^{\alpha(t)}\right)}{(c-x)(c-y)}\right)^{q+1} \sum_{j=0}^{\infty} \frac{\Gamma(q+j+1)}{j ! \Gamma(q+1)}\left(\frac{c^{2} e^{\alpha(t-s)}}{(c-x)(c-y)}\right)^{j}= \\
& =e^{-(x+y) \delta / \gamma}\left(\frac{c^{2}\left(1-e^{\alpha(t-s)}\right)}{(c-x)(c-y)}\right)^{q+1}\left(1-\frac{c^{2} e^{\alpha(t-s)}}{(c-x)(c-y)}\right)^{-(q+1)} .
\end{aligned}
$$

Thus, the obtained expression is equivalent to (43).

The density (40) does not depend on $t$ and its moment generating function is the first cofactor in (42). Because of $\alpha<0$, the parameter $u$ in the second cofactor (42) tends to zero when $(t-s) \rightarrow \infty$, therefore the second cofactor in (42) degenerates in one. Thus 
the density (40) is a probability density of steady-state process of the short interest rate, and the second cofactor in (42) characterizes a transient regime from a initial state of process in at time $s$ to a stationary regime.

It should be noted that one of the Feller's results can be interpreted in our case as follows: if $\widetilde{c}=\beta-\alpha \delta / \gamma>\widetilde{a}=\gamma / 2$, the level $r=-\delta / \gamma$ of the lower reflecting boundary is unattainable. It means that if the inequality $-\beta / \alpha>-\delta / \gamma-\gamma / 2 \alpha$ holds and the short interest rate at some time takes a value exceeding $-\delta / \gamma$, then its values always will exceed the value $-\delta / \gamma$.

Corollary 1 . The probability distribution of the short interest rate process $r(t)$, that is determined by the equation (5) - (7), has the following conditional moments provided that $r(s)=R, s<t$ :

Expectation $E$ :

Variance $V$ :

Correlation coefficient:

Index of skewness:

Index of kurtosis:

$$
\begin{aligned}
& \frac{1+q+u}{c}-\frac{\delta}{\gamma} \\
& \frac{1+q+2 u}{c^{2}} \\
& e^{\alpha(t-s)}
\end{aligned}
$$

$$
\frac{2(1+q+3 u)}{(1+q+2 u)^{3 / 2}}
$$

$$
\frac{3(1+q)(1+q+2(1+2 u))+12 u(2+u)}{(1+q+2 u)^{2}} .
$$

The parameters $u, c$ and $q$ are determined by expressions (38).

Proof implies the calculation of appropriate derivatives of moment generating function (42) at point $\tau=0$ in order to determine the initial moments (or moments about the origin) and then if necessary to recalculate them in central moments (or moments about the mean). To derive the correlation coefficient, the moment generating function (43) is used.

Corollary 2. The moments of random variable that has a marginal probability density (40) are calculated under the formulas:

Expectation $\theta$ :

$$
-\frac{\beta}{\alpha}
$$


Variance $D$ :

$$
\frac{\gamma \beta-\alpha \delta}{2 \alpha^{2}}
$$

Index of skewness:

$$
\gamma \sqrt{\frac{2}{\gamma \beta-\alpha \delta}}
$$

Index of kurtosis:

$$
3+\frac{\gamma^{2}}{\gamma \beta-\alpha \delta}
$$

Proof is the same as in the previous corollary. The formulae of corollary 2 can be obtained also from the formulae of a corollary 1 in the limit $t-s \rightarrow \infty$. In this case $u \rightarrow 0$, and $c \rightarrow-2 \alpha / \gamma(\alpha<0)$. Let's remark that the natural requirement the variance to be positive, i.e. $\gamma \beta>\alpha \delta$, corresponds to the requirement the expectation level to be above than the level of the reflecting boundary, i.e. $-\beta / \alpha>-\delta / \gamma$.

At last we shall remark that the statement of corollary 2 concerns the MC-model, when all four factors of the affine structure in representation of drift and volatility in equalities (6) - (7) can differ from zero. For V-model and CIR-model some coefficients will be reduced to zero and the moments of density (40) for these models are easier expressed:

$\begin{array}{llc} & \text { V-model } & \text { CIR-model } \\ \text { Expectation, } \theta & -\beta / \alpha & -\beta / \alpha \\ \text { Variance, } D & -\delta / 2 \alpha & \gamma \beta / 2 \alpha^{2} \\ \text { Index of skewness } & 0 & \sqrt{2 \gamma / \beta} \\ \text { Index of kurtosis } & 3 & 3+\gamma / \beta\end{array}$

Theorem 3. The conditional probability density of the short interest rate (37) provided that $\gamma \rightarrow 0$ converges to the probability density of normal distribution with expectation $E$ and variance $V$ that are determined by corollary 1 . In addition

$$
\begin{aligned}
& E=R e^{\alpha(t-s)}-\frac{\beta}{\alpha}\left(1-e^{\alpha(t-s)}\right) \quad \text { for any } \gamma \\
& V \underset{\gamma \rightarrow 0}{\longrightarrow} \frac{\delta}{2 \alpha}\left(e^{2 \alpha(t-s)}-1\right) .
\end{aligned}
$$

Proof. The moment generating function of normal distribution for $E$ and $V$ by corollary 1 has the form 


$$
M_{N}(\tau)=\exp \left\{\left(\frac{1+q+u}{c}-\frac{\delta}{\gamma}\right) \tau+\frac{1+q+2 u}{2 c^{2}} \tau^{2}\right\}
$$

To prove the statement it is enough to show that $M_{N}(\tau)$ and the moment generating function $M_{r}(\tau \mid u)$ that is determined by formula (42) provided that $\gamma \rightarrow 0$, have the same limit, that is $\ln _{\gamma \rightarrow 0}\left(M_{N}(\tau) / M_{r}(\tau \mid u)\right)=0$. Let's prove this statement for non-negative $\tau$. The proof of the statement for non-positive $\tau$ is similar. From formula (42) for $0<\tau / c<1$ we have

$$
\begin{aligned}
& \ln M_{r}(\tau \mid u)=\frac{u \tau / c}{1-\tau / c}-\frac{\delta \tau}{\gamma}-(1+q) \ln \left(1-\frac{\tau}{c}\right)= \\
& =u \sum_{j=1}^{\infty}\left(\frac{\tau}{c}\right)^{j}-\frac{\delta \tau}{\gamma}+(1+q) \sum_{j=1}^{\infty} \frac{1}{j}\left(\frac{\tau}{c}\right)^{j}= \\
& =\frac{1+q+u}{c} \tau+\frac{1+q+2 u}{2 c^{2}} \tau^{2}-\frac{\delta \tau}{\gamma}+\sum_{j=3}^{\infty}\left(u+\frac{1+q}{j}\right)\left(\frac{\tau}{c}\right)^{j} .
\end{aligned}
$$

It follows from here that

$$
\ln \frac{M_{N}(\tau)}{M_{r}(\tau)}=-\sum_{j=3}^{\infty}\left(u+\frac{1+q}{j}\right)\left(\frac{\tau}{c}\right)^{j}=-\tau^{2} \sum_{j=1}^{\infty}\left(\frac{u}{c^{2}}+\frac{1+q}{(j+2) c^{2}}\right)\left(\frac{\tau}{c}\right)^{j}
$$

It is not difficult to show that

$$
\frac{u}{c^{2}} \times \frac{\tau}{c-\tau} \leq \sum_{j=1}^{\infty}\left(\frac{u}{c^{2}}+\frac{1+q}{(j+2) c^{2}}\right)\left(\frac{\tau}{c}\right)^{\prime} \leq \frac{1+q+u}{c^{2}} \times \frac{\tau}{c-\tau}
$$

Using explicit expressions of values $u, q$ and $c$ through the model parameters of the short rate by the formulas (38) we shall receive the following limiting relations

$$
\frac{u}{c^{2}}=\frac{\gamma R+\delta}{2 \alpha} e^{\alpha(t-s)}\left(e^{\alpha(t-s)}-1\right) \underset{\gamma \rightarrow 0}{\longrightarrow} \frac{\delta}{2 \alpha} e^{\alpha(t-s)}\left(e^{\alpha(t-s)}-1\right)
$$




$$
\begin{aligned}
& \frac{1+q}{c^{2}}=\frac{\gamma \beta-\alpha \delta}{2 \alpha^{2}}\left(e^{\alpha(t-s)}-1\right)^{2} \underset{\gamma-\gamma 0}{\longrightarrow} \frac{-\delta}{2 \alpha}\left(e^{\alpha(t-s)}-1\right)^{2}, \\
& \frac{\tau}{c-\tau}=\frac{\tau \gamma}{2 \alpha}\left(e^{\alpha(t-s)} 1\right) /\left(1-\frac{\tau \gamma}{2 \alpha}\left(e^{\alpha(t-s)}-1\right)\right) \underset{\gamma \rightarrow 0}{\longrightarrow}>0 .
\end{aligned}
$$

The application of these inequalities and limiting relations in the expression for the logarithm of the ratio of the moment generating functions proves the necessary result $\ln _{r \rightarrow 0}\left(M_{N}(\tau) / M_{r}(\tau \mid u)\right)=0$, that means convergence of the conditional probability density (37) to normal probability density. In summary we shall remark, that expectation $E$ and variance $V$ have the following explicit expressions through the model parameters of the short interest rate

$$
\begin{aligned}
& E=\frac{1+q+u}{c}-\frac{\delta}{\gamma}=R e^{\alpha(t-s)}-\frac{\beta}{\alpha}\left(1-e^{\alpha(t-s)}\right), \\
& V=\frac{1+q+2 u}{c^{2}}=\frac{\gamma \beta-\alpha \delta}{2 \alpha^{2}}\left(e^{\alpha(t-s)}-1\right)^{2}+\frac{\gamma R+\delta}{\alpha} e^{\alpha(t-s)}\left(e^{\alpha(t-s)}-1\right) .
\end{aligned}
$$

Hence, it is seen that $E$ does not depend on $\gamma$, and $V \underset{\gamma \rightarrow 0}{\longrightarrow} \frac{\delta}{2 \alpha}\left(e^{2 \alpha(t-s)}-1\right)$.

Theorem 4 . The joint probability density of the short interest rate (43) provided that $\delta / \gamma \rightarrow \infty$ converges to the probability density of two-dimensional normal distribution with a vector of expectations $(\theta \quad \theta)^{\mathrm{T}}$ and a covariance matrix

$$
\Sigma=\left(\begin{array}{ll}
D & D \rho \\
D \rho & D
\end{array}\right), \quad \rho=e^{a(t-s)},
$$

where $\theta$ and $D$ are determined by the corollary 2 .

Proof. In order to verify validity of this statement, it is enough to show that the moment generating function of the joint probability density (43) provided that $\delta / \gamma \rightarrow \infty$ converges to the moment generating function of two-dimensional normal distribution

$$
M_{N}(u, v)=\exp \left\{\left(\begin{array}{ll}
u & v
\end{array}\right)\left(\begin{array}{l}
\theta \\
\theta
\end{array}\right)+\frac{1}{2}\left(\begin{array}{ll}
u & v
\end{array}\right)\left(\begin{array}{cc}
D & D \rho \\
D \rho & D
\end{array}\right)\left(\begin{array}{l}
u \\
v
\end{array}\right)\right\}=
$$




$$
=\exp \left\{(u+v) \theta+0,5 D\left(u^{2}+2 \rho u v+v^{2}\right)\right\}
$$

Let's for convenience denote $x=\delta / \gamma$. According to the formulae (43), (38) and corollary 2 the moment generating function $M_{r, R}(u, v)$ of the joint probability density can be written as

$$
\begin{aligned}
& M_{r, R}(u, v)=\exp \{-(u+v) x\}\left(\frac{1-\rho}{\left(1-u \frac{D(1-\rho)}{\theta+x}\right)\left(1-v \frac{D(1-\rho)}{\theta+x}\right)-\rho}\right)^{(\theta+x)^{2} / D}= \\
& =\exp \{-(u+v) x\}\left(1-(u+v) \frac{D}{\theta+x}+u v(1-\rho)\left(\frac{D}{\theta+x}\right)^{2}\right)^{-(\theta+x)^{2} / D} .
\end{aligned}
$$

According to the restrictions on the moment generating function (43) this function is determined on a set of variables that satisfies the to an inequality

$$
(u+v) \frac{D}{\theta+x}-u(1-\rho)\left(\frac{D}{\theta+x}\right)^{2}<1 .
$$

It is enough to show that $\ln _{x \rightarrow \infty}\left(M_{N}(u, v) / M_{r, R}(u, v)\right)=0$. For this purpose, first we shall remark that from representation $\ln (1-z)=-\sum_{k=1}^{\infty} z^{k} / k,|\mathrm{z}|<1$, follows that the following incqualitics take place for $0<z<1$

$$
\begin{aligned}
& 0>\ln (1-z)=-z-\frac{z^{2}}{2}-\sum_{k=0}^{\infty} \frac{z^{k}}{k+3}>-z-\frac{z^{2}}{2}-\frac{z^{3}}{1-z}, \\
& 0<\ln (1+z)=z-\frac{z^{2}}{2}+\sum_{k=0}^{\infty} \frac{(-z)^{k}}{k+3}<z-\frac{z^{2}}{2}+\frac{z^{3}}{1-z}
\end{aligned}
$$

Let's now evaluate $\ln M_{r, R}(u, v)$. 


$$
\begin{aligned}
& \ln M_{r, R}(u, v)=-(u+v) x-\frac{(\theta+x)^{2}}{D} \ln \left(1-(u+v) \frac{D}{\theta+x}+u v(1-\rho)\left(\frac{D}{\theta+x}\right)^{2}\right)= \\
& =-(u+v) x+\frac{(\theta+x)^{2}}{D}\left((u+v) \frac{D}{\theta+x}-u v(1-\rho)\left(\frac{D}{\theta+x}\right)^{2}\right)+ \\
& +\frac{(\theta+x)^{2}}{2 D}\left((u+v) \frac{D}{\theta+x}-u v(1-\rho)\left(\frac{D}{\theta+x}\right)^{2}\right)^{2}+ \\
& +\frac{(\theta+x)^{2}}{D} \sum_{k=3}^{\infty} \frac{1}{k}\left(\frac{D}{\theta+x}\right)^{k}\left(u+v-u v(1-\rho)\left(\frac{D}{\theta+x}\right)\right)^{k}= \\
& \quad=(u+v) \theta+\frac{1}{2} D\left(u^{2}+2 \rho u v+v^{2}\right)-u v(u+v)(1-\rho) \frac{D}{\theta+x}+ \\
& +\frac{1}{2}(u v)^{2}(1-\rho)^{2} D\left(\frac{D}{\theta+x}\right)^{2}+D \sum_{k=3}^{\infty} \frac{1}{k}\left(\frac{D}{\theta+x}\right)^{k-2}\left(u+v-u v(1-\rho)\left(\frac{D}{\theta+x}\right)\right)^{k} .
\end{aligned}
$$

Therefore

$$
\begin{aligned}
& \ln \frac{M_{N}(u, v)}{M_{r, R}(u, v)}=2 u v(u+v)(1-\rho) \frac{D}{\theta+x}-\frac{1}{2}(u v)^{2}(1-\rho)^{2} D\left(\frac{D}{\theta+x}\right)^{2}- \\
& -D \sum_{k=1}^{\infty} \frac{1}{k+2}\left(\frac{D}{\theta+x}\right)^{k}\left(u+v-u v(1-\rho)\left(\frac{D}{\theta+x}\right)\right)^{k+2}=O\left(\frac{D}{\theta+x}\right)
\end{aligned}
$$

From here it also follows that

$$
\lim _{x \rightarrow \infty} \ln \frac{M_{N}(u, v)}{M_{r, R}(u, v)}=0,
$$

that is the validity of the statement of the theorem 4 . 
Theorem 5. The probability density of the short interest rate (40) provided that $\delta / \gamma \rightarrow \infty$ converges to the probability density of normal distribution with expectation $\theta$ and variance $D$ that are determined by corollary 2 .

Proof. It is enough to show that the moment generating function of the probability density of the short interest rate (40) provided that $\delta / \gamma \rightarrow \infty$ converges to the moment generating function of normal distribution

$$
M_{N}(\tau)=\exp \left\{\theta \tau+\frac{1}{2} D \tau^{2}\right\}
$$

The moment generating function of the probability density (40) is expressed as (44). Because $M_{r}(\tau)$ is the moment generating function of shifted gamma distribution, the following relations take place between expectation $\theta$, variance $D$ and parameters of this distribution $q, c_{0}, x=\delta / \gamma$

$$
\theta=\frac{q+1}{c_{0}}-\frac{\delta}{\gamma}, D=\frac{q+1}{c_{0}^{2}}, c_{0}=\frac{\theta+x}{D}, q+1=\frac{(\theta+x)^{2}}{D} .
$$

The moment generating function $M_{r}(\tau)$ can be written through $\theta$ and $D$ as follows

$$
M_{r}(\tau)=e^{-x \tau}\left(1-\frac{D \tau}{\theta+x}\right)^{-\frac{(\theta+x)^{2}}{D}}, \quad \tau<\frac{\theta+x}{D}
$$

Let's consider logarithm of the ratio $M_{N}(\tau) / M_{r}(\tau)$

$$
\begin{aligned}
& \ln \frac{M_{N}(\tau)}{M_{r}(\tau)}=\theta \tau+\frac{1}{2} D \tau^{2}+x \tau+\frac{(\theta+x)^{2}}{D} \ln \left(1-\frac{D \tau}{\theta+x}\right)= \\
& =(\theta+x) \tau+\frac{D \tau^{2}}{2}+\frac{(\theta+x)^{2}}{D}\left(-\frac{D \tau}{\theta+x}-\frac{1}{2}\left(\frac{D \tau}{\theta+x}\right)^{2}-\frac{1}{3}\left(\frac{D \tau}{\theta+x}\right)^{3}-\ldots\right)= \\
& =-\frac{1}{3} \frac{D \tau^{3}}{\theta+x}\left(1+\frac{3}{4}\left(\frac{D \tau}{\theta+x}\right)+\frac{3}{5}\left(\frac{D \tau}{\theta+x}\right)^{2}+\ldots\right) .
\end{aligned}
$$


The sum of a series in brackets is easily evaluated from above and from below. Let's do it only for positive $\tau$. For negative $\tau$ it is similar.

$$
\frac{2}{2-\frac{D \tau}{\theta+x}}<1+\frac{3}{4}\left(\frac{D \tau}{\theta+x}\right)+\frac{3}{5}\left(\frac{D \tau}{\theta+x}\right)^{2}+\ldots<\frac{1}{1-\frac{D \tau}{\theta+x}}
$$

Therefore

$$
-\frac{D \tau^{3}}{3(\theta+x-D \tau)}<\ln \frac{M_{N}(\tau)}{M_{r}(\tau)}<-\frac{2 D \tau^{3}}{6(\theta+x)-3 D \tau}
$$

From here it follows that provided that $\delta / \gamma \rightarrow \infty$ the convergence $\ln \frac{M_{N}(\tau)}{M_{r}(\tau)} \rightarrow 0$ takes place, or $\lim _{\delta / \gamma \rightarrow \infty} M_{r}(\tau)=M_{N}(\tau)$ as it was needed to show.

The theorems in this section have been proved for the MC-model. From these theorems we conclude that the reflecting boundary $-\delta / \gamma$ has the very strong influence on the kind of the probability density of the short interest rate $r(t)$. In general case this probability density is a shifted gamma density (40). If $\delta / \gamma=0(\delta=0, \gamma>0)$ then the probability density of the short interest rate $r(t)$ is a classical gamma density. In this case the MC-model is converted in the CIR-model. At last if the reflecting boundary $-\delta / \gamma \rightarrow$ $-\infty$ (i.e. $\delta>0, \gamma \rightarrow 0$ ) and the expectation $\theta$ and the variance $D$ are fixed then the probability density of the short interest rate $r(t)$ converges to the density of normal distribution and the MC-model is converted in the V-model.

It can seen that in general, the model with the negative reflecting boundary can not be realistic. However it is possible that such a model will be more likelihood for some real sample data. Indeed if some model with a negative reflecting boundary will be rather exactly describing a real process with very small probability of negative interest rate values, then it seems such a model can be used in the practice (Rogers, 1995). The final decision about such problem can be taken using the statistical analysis.

Conclusions

As it can be seen from the literature (Björk, 1995; Brown and Schaefer, 1994; Duffie, 1992; Rogers, 1995; Vetzal, 1994) the affine yield class of the term structure is very important for obtaining the fundamental properties of the zero-coupon bond yield. 
Therefore any new results about the properties the affine yield class are very desirable. The present paper is devoted to this problem. The main results of the paper are following.

The lemma of the Introduction determines the solution of the Riccati equation (9) for the function of the affine term structure $B(t, T)$ when the coefficients of the affine structure are constant or are connected by relation (12).

If the coefficients of the affine structure are constant rather simple integral relation (20) is derived for the function of the affine term structure $A(t, T)$. These results allow us to obtain some equivalent explicit expressions for function $A(t, T)$ under assumptions of the MC-model and as special cases for the V-model and the CIR-model.

The propositions $1-3$ of the Section 1 explain the properties of the functions of the affine term structure $A(t, T)$ and $B(t, T)$ provided that the coefficients of affine structure are constant and give the comparative characteristics of these functions for the $\mathrm{V}$-model, the CIR-model and the MC-model.

The theorem 1 of the Section 2 set the explicit forms for the conditional probability density, the joint probability density and the marginal probability density of the short interest rate $r(t)$ under assumptions of the MC-model. In general case, the marginal probability density of the short interest rate is a shifted gamma density. The conditional probability density is a mixture of the shifted gamma densities with different shape parameters. The mixing variable is a Poisson random variable with Poisson parameter that is directly proportional to the value of short interest rate at time of condition. The joint probability density is a mixture of pairs of identical shifted gamma densities with different shape parameters. But the mixing variable in this case is a random variable with a negative binomial probability distribution. For the proof of these results the Feller's technique has been essentially used.

Let us understand the structure of conditional and marginal probability densities of the short interest rate as the probability structure of this interest rate process. In stationary regime this stochastic process $r(t)$ has the shifted gamma density. In transient regime when the value of short interest rate $r(t)$ is dependent on given value $r(s), s<t$, the value $r(t)$ can be present as a sum of two components. The first component has shifted gamma density as in the stationary regime. The second component has compound Poisson distribution, i.e. it is a random sum of the exponentially distributed random variables with parameter that is equal to 1 . The number of terms of the random sum is a random variable with the Poisson distribution. The Poisson parameter of this distribution depends on the length of the time interval $(s, t)$ and tends to zero provided that $(t-s) \rightarrow \infty$. It means that if $(t-s) \rightarrow \infty$ then the second component converges to zero, too.

The explicit forms of moment generating functions for conditional, joint and marginal probability densities of the short interest rate are set by theorem 2 . It is seen from theorem this that the explicit forms of moment generating functions have more simple structures than appropriate probability densities. It allows us, for example, very simply to analyze the transient regime of short interest rate process by moment generating function 
(42). The corollaries 1 and 2 set the leading four conditional and marginal moments of the short interest rate.

The simple structure of moment generating functions makes possible easily to prove the limit theorems $3-5$ concerning the convergence of the conditional, joint and marginal probability densities of the short interest rate to the probability densities of normal distribution provided that the low reflecting boundary $-\delta / \gamma$ tends to $-\infty$.

In the framework of the affine yield class of the term structure, the price of the zerocoupon bonds is determined by relation (1) in terms of the short interest rate $r$ and the functions of the affine term structure $A(t, T)$ and $B(t, T)$. Therefore, the present paper is devoted to the analysis of the properties of $r(t), A(t, T)$ and $B(t, T)$. However, the short interest rate process $r(t)$ is unobservable in the practice. Hence, the values of the short interest rate process can not be used for the identification of the adequate model bond price process. At the same time it is known that bid quotations on the most actively traded securities on the market are base for issuing the yield curves. Therefore, from the practical point of view it is more natural to use the yield interest rate (2) instead of short interest rate $r(t)$. Thus it is interesting problem to identify the adequate model bond price process by handling of yield rate samples. The solution of this problem will allow us to understand which of considered above models is more relevant to describe the bond price process. We leave this problem to a next paper.

\section{References}

Björk, T. (1995). Arbitrage theory in continuous time. Stockholm: Stockholm School of Economics.

Brown, R. H., S. M. Schaefer. (1991). Interest rate volatility and the term structure. Working paper, London Business School, London.

Brown, R. H., S. M. Schaefer. (1994). Interest rate volatility and the shape of the term structure. Phil. Trans. R. Soc. Lond. Vol. A 347. P. 563-576.

Cox J., Ingersoll J., Ross S. (1985). A theory of the term structure of interest rates. Econometrica. Vol. 53, № 2. P. 385-407.

Duffie, D. (1992). Dynamic asset pricing theory. Princeton: Princeton University Press.

Duffie, D., R. Kan. (1993). A yield-factor model of interest rates. Working paper, Stanford University, Stanford.

Feller W. (1951). Two singular diffusion problems. Annals of Mathematics. Vol. 54, No. 1. P. 173-182.

Gerber, H. U. (1979). An introduction to mathematical risk theory. Philadelphia: University of Pennsylvania.

Kamke E. (1959). Differentialgleichungen. Lusungsmethoden und lusungen. Vol. 1. Gewuhnliche differentialgleichungen. Leipzig. 
Lamperti, J. (1977). Stochastic processes. A survey of the mathematical theory. New York: Springer-Verlag.

Medvedev G. A., Cox S. H. (1996). The market price of risk for affine interest rate term structures. Proceedings of the 6-th International AFIR Symposium. Nuremberg. P. 913-924.

Rogers L.C.G. (1995). Which model for term-structure of interest rates should one use? Mathematical Finance (ed. M. Davis et al.). New York: Springer-Verlag. P. 93-115.

Vasiček O. (1977). An equilibrium characterization of the term structure. Journal of Financial Economics. Vol. 5. P. 177-188.

Vetzal K. R. (1994). A survey of stochastic continuous time models of the term structure of interest rates. Insurance: Mathematics and Economics. Vol. 14. P. 139-161. 
